\title{
ON THE DIMENSIONS OF SECTIONS FOR THE GRAPH-DIRECTED SETS
}

\author{
Zhi-Ying Wen and Li-Feng $\mathrm{Xi}^{*}$ \\ Tsinghua University, Department of Mathematics \\ 10084, Beijing, P. R. China; wenzy@tsinghua.edu.cn \\ Zhejiang Wanli University, Institute of Mathematics \\ 315100, Ningbo, Zhejiang, P. R. China; xilifengningbo@yahoo.com
}

\begin{abstract}
The various dimensions of the intersections of the graph-directed sets $\left\{K_{i}\right\}_{i=1}^{l} \subset \mathbf{R}^{n}$ with $(n-m)$-planes $V+a_{i}\left(a_{i} \in V^{\perp}\right)$ were investigated for $\mathscr{H}^{m}$ almost all parameters $a_{i} \in V^{\perp}$ satisfying $\left(V+a_{i}\right) \cap K_{i} \neq \varnothing$, where $V \subset \mathbf{R}^{n}$ is a fixed $(n-m)$-dimensional subspace and $V^{\perp}$ its orthogonal complement. We obtain the typical value of dimensions of sections for typical directions $V$ and also provide a weaker result for exceptional directions.
\end{abstract}

\section{Introduction}

1.1. Graph-directed construction. Let $m$ and $n$ be integers with $0<m<n$. Denote by $O(n)$ the orthogonal transformation group of $\mathbf{R}^{n}$. Given $\rho \in(0,1), b \in \mathbf{R}^{n}$ and $R \in O(n)$, we obtain a contracting similitude $S$ of $\mathbf{R}^{n}$ defined by $S(x)=\rho R x+b$.

We recall the graph-directed construction [MW] as follows: Suppose $G$ is a directed graph, which contains $l$ vertexes $\{1, \cdots, l\}$ and some directed edges $\{e: e \in G\}$ among the vertexes. Let $\Gamma_{i, j}$ be the set of all the edges from $i$ to $j$. Assume $\left\{K_{1}, \cdots, K_{l}\right\}$ is a family of compact sets in $\mathbf{R}^{n}$, and there are similitudes $\left\{S_{e}: e \in G\right\}$ such that

$$
K_{i}=\bigcup_{j} \bigcup_{e \in \Gamma_{i, j}} S_{e}\left(K_{j}\right)
$$

where $S_{e}(x)=\rho_{e} R_{e}(x)+b_{e}$ with $\rho_{e} \in(0,1), R_{e} \in O(n)$ and $b_{e} \in \mathbf{R}^{n}$. We say that $G$ is irreducible, if for any vertex pair $(i, j)$ there exists an admissible directed path starting at $i$ and ending at $j$.

For any admissible directed path $e^{*}=e_{1} \cdots e_{k}$ in $G$, write $S_{e^{*}}=S_{e_{1} \cdots e_{k}}=$ $S_{e_{1}} \circ \cdots \circ S_{e_{k}}, \rho_{e^{*}}=\rho_{e_{1}} \cdots \rho_{e_{k}}$ and $R_{e^{*}}=R_{e_{1}} \circ \cdots \circ R_{e_{k}}$. Let

$$
A_{i, j}=\left\{R_{e^{*}}: e^{*}=e_{1} e_{2} \cdots e_{k} \text { is a path from } i \text { to } j\right\} \subset O(n) .
$$

To ensure certain finiteness, we pose the following assumption:

$$
G \text { is irreducible and } \# A_{i, j}<\infty \text { for some } 1 \leq i, j \leq l \text {. }
$$

Under this assumption of irreducibility, the Hausdorff dimensions of $K_{1}, \cdots, K_{l}$ have the same value, denoted by $s$.

The structure of the sets $A_{i, j}$ is described in the following lemma.

doi:10.5186/aasfm.2010.3532

2000 Mathematics Subject Classification: Primary 28A80.

Key words: Graph-directed set, dimension, plane section.

* Corresponding author. Supported by the National Natural Science Foundation of China (Grant Nos. 10671180, 10571104, 10631040), National Basic Research Program of China (973 Program) 2007CB814800 and partially by the Morningside Center of Mathematics in Beijing. 

$l$,

Lemma 1. Suppose $G$ is irreducible and $\# A_{i_{0}, j_{0}}<\infty$. Then for any $1 \leq i, j, k \leq$

(1) $\# A_{i, j}=\# A_{i_{0}, j_{0}}$;

(2) $A_{i, j}=A_{i, k} A_{k, j}$;

(3) $A_{i, i}$ is a finite subgroup of $O(n)$ for any $i$.

Proof. (1) By using irreducibility, take any $h \in A_{i_{0}, i}, t \in A_{j, j_{0}}$. Then $h A_{i, j} t \subset$ $A_{i_{0}, j_{0}}$, which implies that $\# A_{i, j}=\#\left(h A_{i, j} t\right) \leq \# A_{i_{0}, j_{0}}<\infty$ since $t, h \in O(n)$ are invertible. On the other hand, by using irreducibility again, take $u \in A_{i, i_{0}}$ and $v \in A_{j_{0}, j}$, and we have $u A_{i_{0}, j_{0}} v \subset A_{i, j}$, which implies $\# A_{i_{0}, j_{0}} \leq \# A_{i, j}$. Therefore, $\# A_{i j}=\# A_{i_{0}, j_{0}}$.

(2) Take any $g \in A_{k, j}$; then $A_{i, k} g \subset A_{i, j}$. Since $\#\left(A_{i, k} g\right)=\# A_{i, k}=\# A_{i, j}$, then $A_{i, k} g=A_{i, j}$. On the other hand, it follows from the definition that $A_{i, k} A_{k, j} \subset A_{i, j}$. Therefore, $A_{i, k} A_{k, j}=A_{i, j}$.

(3) Since $A_{i, i} A_{i, i} \subset A_{i, i}$ and $\# A_{i, i}<\infty, A_{i i}$ is a finite subgroup of $O(n)$.

The following examples illustrate condition (1.2).

Example 1. Let $S_{i}(x)=\rho_{i} R_{i}(x)+b_{i}$ for $1 \leq i \leq k$. If

$$
\left\{R_{i}\right\}_{i} \text { is contained in a finite subgroup of } O(n) \text {, }
$$

then assumption (1.2) holds.

Scaling self-similar set: If $R_{i}$ is the identical mapping for each $i$, then the invariant set of the similitudes

$$
F_{i}(x)=\rho_{i} x+b_{i}
$$

is said to be a scaling self-similar set, e.g., the Sierpinski carpet.
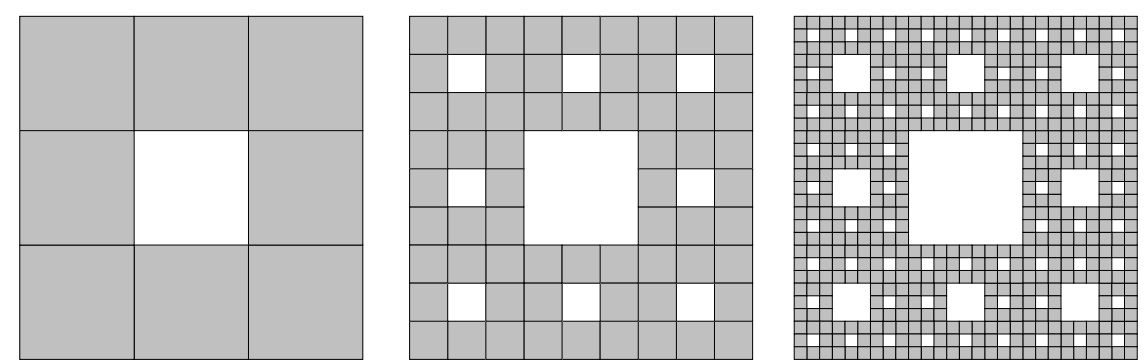

Figure 1. Steps of generating the Sierpinski carpet.

For the Koch curve, the orthogonal transformations of its corresponding similitudes are contained in a finite rotation group $\left\{e^{i k \pi / 3}: k \in \mathbf{Z}\right\}$, and thus assumption (1.3) holds.

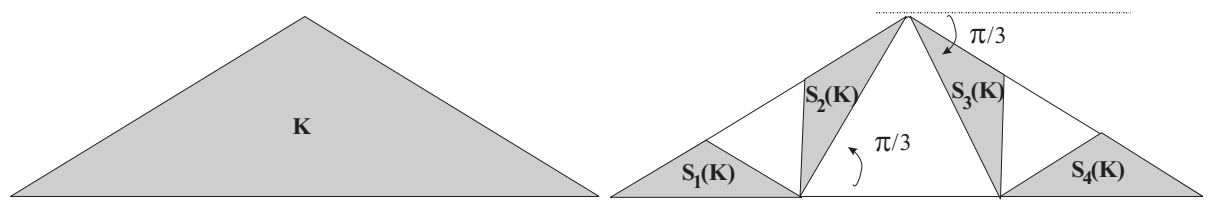

Figure 2. Steps of generating the Koch curve. 

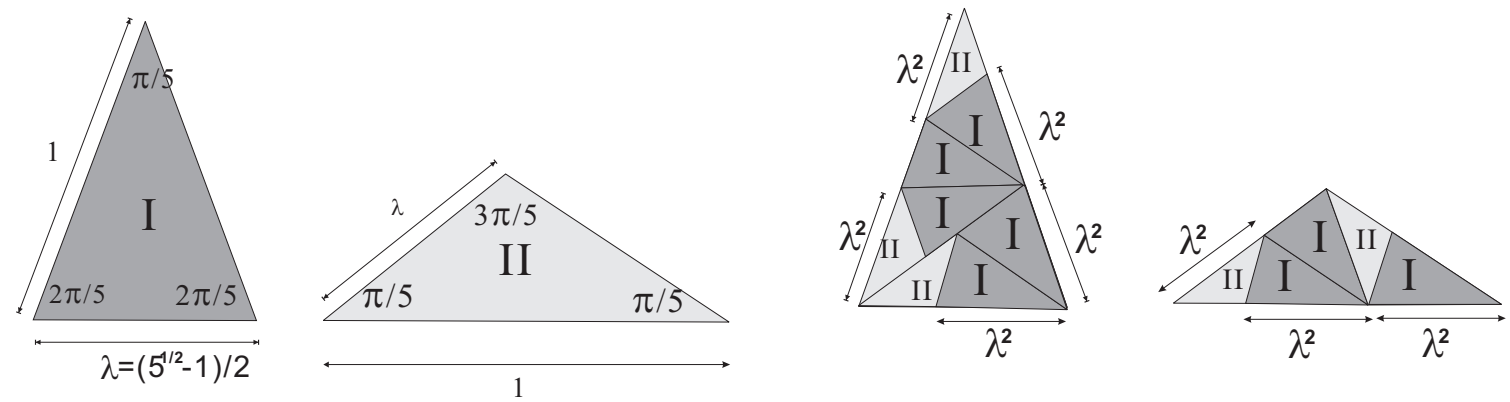

Figure 3. Penrose tiling: a graph-directed construction.

Example 2. In Figure 3, solid triangles I and II include several copies with ratio $\lambda^{2}\left(\lambda=\frac{\sqrt{5}-1}{2}\right)$ of themselves, respectively. Keep some selected copies as the initial pattern and replace them by smaller contained copies of elements in the pattern, continue this procedure over and over again, and we always get two limit sets, named Penrose fractals, with graph-directed construction. Here we suppose that the graph-directed construction is irreducible. Notice that any rotation appearing in the corresponding similitudes belongs to $\left\{e^{i k \pi / 5}: k \in \mathbf{Z}\right\}$, and thus (1.2) holds.

1.2. Dimensions of sections. For any $(n-m)$-dimensional subspace $V \subset \mathbf{R}^{n}$, let $V^{\perp}=\left\{x \in \mathbf{R}^{n}: x \perp V\right\}$ be its orthogonal complement. Given $z \in \mathbf{R}^{n}$, let $V+z$ denote the $(n-m)$-plane $\{y+z: y \in V\}$. For $a \in V^{\perp}$, we consider $K_{i} \cap(V+a)$, the intersections of graph-directed sets $K_{1}, \cdots, K_{l}$ with the $(n-m)$-plane $V+a$. Denote by $J_{V, i}$ the set of all the parameters $a \in V^{\perp}$ such that $K_{i} \cap(V+a)$ is non-empty, that is to say

$$
J_{V, i}=\left\{a \in V^{\perp}: K_{i} \cap(V+a) \neq \varnothing\right\}=P_{V^{\perp}}\left(K_{i}\right),
$$

where $P_{V^{\perp}}: \mathbf{R}^{n} \rightarrow V^{\perp}$ is the corresponding orthogonal projection. Notice that if $s>m$, then $\mathscr{H}^{m}\left[P_{V^{\perp}}\left(K_{i}\right)\right]>0$ for $\gamma_{n, m}$ almost all $V^{\perp} \in G(n, m)$, where $\mathscr{H}^{m}$ is the $m$-dimensional Hausdorff measure on $V^{\perp}$. Here $G(n, m)$ is the Grassmannian manifold consisting of all $m$-dimensional linear subspaces of $\mathbf{R}^{n}$, and $\gamma_{n, m}$ is the natural measure on it such that $\gamma_{n, m}(A)=\alpha^{-m}(n)\left(\mathscr{L}^{n} \times \cdots \times \mathscr{L}^{n}\right)\left(\left\{\left(v_{1}, \cdots v_{m}\right) \in\right.\right.$ $\left(\mathbf{R}^{n}\right)^{m},\left|v_{i}\right| \leq 1$ for all $i$ and $\left.\left.L\left(v_{1}, \cdots, v_{m}\right) \in A\right\}\right)$, where $L\left(v_{1}, \cdots, v_{m}\right)$ is the subspace spanned by the vector $v_{1}, \cdots, v_{m} \in \mathbf{R}^{n}$ [Mat5].

In the paper, we will investigate the various dimensions of the plane section $(V+a) \cap K_{i}$ for $\mathscr{H}^{m}$ almost all $a \in P_{V^{\perp}}\left(K_{i}\right)$.

Recall that there are plenty of achievements on the dimensions of plane sections or the measures supported on sections. Among these, the following Marstrand's theorem [M, Mat2] is well-known: Suppose $m<s<n$, and $A \subset \mathbf{R}^{n}$ is a Borel set with $0<\mathscr{H}^{s}(A)<\infty$. Then

(1) for $\gamma_{n, n-m}$-almost all $V \in G(n, n-m)$,

$$
\mathscr{H}^{m}\left\{a \in V^{\perp}: \operatorname{dim}_{H}[A \cap(V+a)]=s-m\right\}>0 ;
$$

(2) for $\mathscr{H}^{s} \times \gamma_{n, n-m}$-almost all $(x, V) \in A \times G(n, n-m)$,

$$
\operatorname{dim}_{H}[A \cap(V+x)]=s-m, \mathscr{H}^{s-m}[A \cap(V+x)]<\infty .
$$

In fact, for $n=2, m=1,(1)$ and (2) were first proved by Marstrand [M] and later generalized by Mattila [Mat1] to higher dimensions. Furthermore, the intersections $A \cap f B$ were researched for given compact sets $A, B \subset \mathbf{R}^{n}$, where $f$ runs through the isometry group or other geometric transformation groups of $\mathbf{R}^{n}$ [Mat4, K]. 
The packing dimension of plane sections with corresponding measures was discussed in [C, FJM, FJ, FM, JM], and so on. They showed that Marstrand's theorem is not valid for the packing dimension. For example, in [FJM] Falconer, Järvenpää and Mattila gave some examples illustrating the instability of packing dimensions of sections. Moreover, in [C] Csörnyei obtained a planar construction which allows one to prescribe the packing dimensions of line sections, that is, given a Borel measurable function $f$ from the space of planar lines into [0,1], there is a Borel set $A \subset \mathbf{R}^{2}$ such that for a.e. line $l$,

$$
\operatorname{dim}_{P}(A \cap l)=f(l) .
$$

In $[\mathrm{M}]$ and [Mat1], the direction $V$ is random. Notice that in [BP] and [KP], Benjamini, Kenyon and Peres studied the intersections of some special planar sets with lines in a fixed direction. For example, in [BP] the dimensions of fibres $F_{x}=$ $\{y \in[0,1]:(x, y) \in F\}$ for almost all $x \in[0,1]$ were discussed for some certain geometric construction in the unit square $[0,1] \times[0,1]$.

1.3. Typical cases: Theorem 1. Under the above notations, we will state our first result on typical parameters as follows (see Theorem 2 for the exceptional case):

Theorem 1. Suppose $\left\{K_{1}, \cdots, K_{l}\right\}$ are graph-directed sets satisfying (1.1) and (1.2). Assume $\operatorname{dim}_{H} K_{1}=\cdots=\operatorname{dim}_{H} K_{i}=s \geq m$. Then for each $1 \leq i \leq l$, $\gamma_{n, n-m}$-almost all $V \in G(n, n-m)$, and $\mathscr{H}^{m}$-almost all $a \in P_{V^{\perp}}\left(K_{i}\right)$,

$$
\begin{aligned}
\operatorname{dim}_{H}\left[(V+a) \cap K_{i}\right] & =\underline{\operatorname{dim}}_{B}\left[(V+a) \cap K_{i}\right]=\overline{\operatorname{dim}}_{B}\left[(V+a) \cap K_{i}\right] \\
& =\operatorname{dim}_{P}\left[(V+a) \cap K_{i}\right]=s-m .
\end{aligned}
$$

As the self-similar structure is a special irreducible graph-directed construction, we have the following corollary:

Corollary 1. Given similitudes $S_{i}(x)=\rho_{i} R_{i}(x)+b_{i}(1 \leq i \leq k)$, let $E$ denote the self-similar set generated by $\left\{S_{i}\right\}_{i}$. Suppose that the set

$$
\left\{R_{i}\right\}_{i} \text { is contained in a finite subgroup of } O(n) \text {. }
$$

Then assumption (1.2) holds. If $\operatorname{dim}_{H} E>m$, then for $\gamma_{n, n-m}$-almost all $V \in$ $G(n, n-m)$, and $\mathscr{H}^{m}$-almost all $a \in P_{V^{\perp}}(E)$,

$$
\operatorname{dim}_{H} E_{V, a}=\underline{\operatorname{dim}}_{B} E_{V, a}=\overline{\operatorname{dim}}_{B} E_{V, a}=\operatorname{dim}_{P} E_{V, a}=\operatorname{dim}_{H} E-m,
$$

where $E_{V, a}=(V+a) \cap E$.

Remark 1. In this remark, for notational convenience, we only discuss Theorem 1 for self-similar sets. By Marstrand's theorem, for the general compact set $E$, we only have

$$
\mathscr{H}^{m}\left(C_{V}\right)>0
$$

where $C_{V}=\left\{a \in V^{\perp}: \operatorname{dim}_{H}[E \cap(V+a)]=\operatorname{dim}_{H} E-m\right\} \subset J_{V}=P_{V^{\perp}} E$, and we cannot obtain the conclusion that

$$
C_{V} \text { has full measure } \mathscr{H}^{m}\left(J_{V}\right) \text { for a.e. direction } V \text {, }
$$

as shown in Theorem 1 for the self-similar sets.

In the following Example 3, $E$ is not a self-similar set and there is $\Lambda \subset G(n, n-m)$ with $\gamma_{n, n-m}(\Lambda)>0$ such that

$$
\mathscr{H}^{m}\left(C_{V}\right)<\mathscr{H}^{m}\left(J_{V}\right) \text { whenever } V \in \Lambda \text {. }
$$

That means for the general compact sets not self-similar, 
(1) Marstrand's theorem is sharp, that is, (1.5) is false,

(2) and Theorem 1 is invalid.

Example 3. Suppose that $A$ and $B$ are two self-similar sets, generated by contractions in the form of (1.4), satisfying

$$
m<\operatorname{dim}_{H} A<\operatorname{dim}_{H} B .
$$

Assume that the least distance between $A$ and $B$ is so large that there exists a set $\Lambda \subset G(n, n-m)$ with $\gamma_{n, n-m}(\Lambda)>0$ such that for any $V \in \Lambda$, the sets $P_{V^{\perp}}(A)$, $P_{V^{\perp}}(B)$ are disjoint and $\mathscr{H}^{m}\left(P_{V^{\perp}} A\right)>0$.

Let $E=A \cup B$; then $\operatorname{dim}_{H} E=\operatorname{dim}_{H} B$. For any $V \in \Lambda$ and $a \in P_{V^{\perp}}(A)$, $(V+a) \cap E \subset A$, which means $(V+a) \cap E=(V+a) \cap A$. By Theorem 1 , the section $(V+a) \cap E$ has dimension

$$
\operatorname{dim}_{H} A-m\left(<\operatorname{dim}_{H} E-m\right)
$$

for $\mathscr{H}^{m}$ almost all $a \in P_{V^{\perp}}(A)$. Here $\mathscr{H}^{m}\left(P_{V^{\perp}} A\right)>0$ and $P_{V^{\perp}} A \subset J_{V}=P_{V^{\perp}}(E)$.

Example 4. The Sierpinski carpet $E$ has dimension $\log 8 / \log 3$. Let $L_{\theta, b}=$ $\{(x, y): y=(\tan \theta) x+b\}$ and $J_{\theta}=\left\{b: E \cap L_{\theta, b} \neq \varnothing\right\}$. Then

$$
J_{\theta}= \begin{cases}{[-\tan \theta, 1]} & \text { if } \theta \in(0, \pi / 2), \\ {[0,1-\tan \theta]} & \text { if } \theta \in(\pi / 2, \pi),\end{cases}
$$

is an interval.

(1) Then by Theorem 1 , there is a small direction set $D \subset(0,2 \pi)$ with $\mathscr{H}^{1}(D)=$ 0 such that given any $\theta \in(0,2 \pi) \backslash D$, for $\mathscr{H}^{1}$-almost all $b \in J_{\theta}$,

$$
\operatorname{dim}_{H} E \cap L_{\theta, b}=\log 8 / \log 3-1,
$$

where $\operatorname{dim}_{H}$ can be replaced with $\operatorname{dim}_{B}$ or $\operatorname{dim}_{P}$. But from Marstrand's Theorem, we only have

$$
\mathscr{L}^{1}\left\{b: \operatorname{dim}_{H} E \cap L_{\theta, b}=\log 8 / \log 3-1\right\}>0 .
$$

(2) For $\tan \theta \in \mathbf{Q}$, by [LXZ], $\operatorname{dim}_{H} E \cap L_{\theta, b}=c_{\theta}$ for a.e. $b \in J_{\theta}$. For example, $\theta=\pi / 4, c_{\theta}=0.8858 \cdots<\log 8 / \log 3-1$ (see also $[\mathrm{KP}]$ for $\tan \theta=1$ ).

(3) When $b$ and $\tan \theta \in \mathbf{Q}$, as shown in [L], the section $E \cap L_{\theta, b}$ will generate a graph-directed construction, and thus its dimension can be computed.

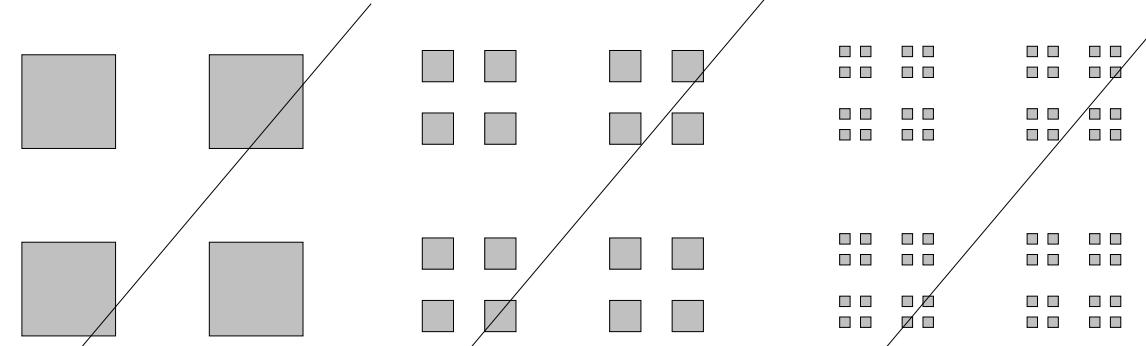

Figure 4. The first three steps of generating $C \times C$.

Example 5. Let $F=C \times C$, where $C$ is the Cantor ternary set. Then $\operatorname{dim}_{H} F=$ $\log 4 / \log 3$. Let the line $L_{\theta, b}=\{(x, y): y=(\tan \theta) x+b\}$, and $\mathscr{J}_{\theta}=\left\{b: F \cap L_{\theta, b} \neq \varnothing\right\}$. 
Then by Theorem 1 , there is a small set $\mathscr{D} \subset(0,2 \pi)$ with $\mathscr{H}^{1}(\mathscr{D})=0$ such that given any $\theta \in(0,2 \pi) \backslash \mathscr{D}$, for $\mathscr{H}^{1}$-almost all $b \in \mathscr{J}_{\theta}$,

$$
\operatorname{dim}_{H} F \cap L_{\theta, b}=\log 4 / \log 3-1,
$$

where $\operatorname{dim}_{H}$ could be $\operatorname{dim}_{B}$ or $\operatorname{dim}_{P}$.

Here (1.6) is true for any typical direction, but it is invalid even for $\theta_{0}=\pi / 4$. In fact, for almost all $b \in \mathscr{J}_{\theta_{0}}=[-1,1], \operatorname{dim} F \cap L_{\theta_{0}, b}=\log 2 /(3 \log 3)$. That is a consequence of the following result of Hawkes [Ha]:

$$
\operatorname{dim}[C \cap(C+t)]=\log 2 /(3 \log 3) \text { for } \mathscr{H}^{1} \text { a.e. } t \in[-1,1] .
$$

where $\log 2 /(3 \log 3)<\log 4 / \log 3-1$.

In addition, when $b$ and $\tan \theta \in \mathbf{Q}$, as shown in [L], the section $F \cap L_{\theta, b}$ will generate a graph-directed construction and thus its dimension can be computed. For example, let $G$ denote the corresponding line section with respect to the line $y=2 x / 5$. Then

$$
\operatorname{dim}_{H} G=\operatorname{dim}_{B} G=\operatorname{dim}_{P} G=0.34793 \cdots .
$$

Example 6. Given $\left\{\rho_{i}\right\}_{i=1}^{k}$ satisfying $0<\rho_{i}<1$ and $\sum_{i=1}^{k}\left(\rho_{i}\right)^{s}=1$ with $s>$ $m(\in \mathbf{N})$. Let $E(c) \subset \mathbf{R}^{n}$ be the self-similar set generated by $T_{i} x=\rho_{i} x+c_{i}: \mathbf{R}^{n} \rightarrow \mathbf{R}^{n}$ $(1 \leq i \leq k)$, where $c=\left(c_{1}, \cdots, c_{k}\right) \in\left(\mathbf{R}^{n}\right)^{k}$. Let $J_{V}(c)=\left\{a \in V^{\perp}: E(c) \cap(V+a) \neq\right.$ $\varnothing\}$. By Theorem 9.12 in [Fa2], just for the simple case of similitudes, for $\left(\mathscr{H}^{n}\right)^{k}$ almost all $\left(c_{1}, \cdots c_{k}\right) \in\left(\mathbf{R}^{n}\right)^{k}$,

$$
\operatorname{dim}_{H} E(c)=s .
$$

It follows from Theorem 1 that for $\gamma_{n, n-m}$ almost all $V \in G(n, n-m), \mathscr{H}^{m}\left(J_{V}(c)\right)>0$ and for any dimension dim appearing in Theorem 1 ,

$$
\operatorname{dim}[E(c) \cap(V+a)]=s-m .
$$

for $\mathscr{H}^{m}$ almost all $a \in J_{V}(c)$.

Example 7. The Koch curve $H$ has dimension $\log 4 / \log 3>1$. Then by Corollary 1 , for a typical direction $V=\{(x, y): y=(\tan \theta) x\}$ and a typical parameter $a \in J_{V}$ (an interval), the section $(V+a) \cap H$ has dimension $\log 4 / \log 3-1$.

Example 8. In Figure 5, solid rectangles I and II include several copies with ratio $1 / 2$ of themselves, respectively. Any related similitude has the scaling form, $S_{e}(x)=\rho_{e} x+b_{e}$, and thus (1.2) holds. The limit sets have dimension $\log 3 / \log 2$. As the limit sets are path-connected, their projections are intervals. Then for typical parameters, the corresponding section has dimension $\log 3 / \log 2-1$.

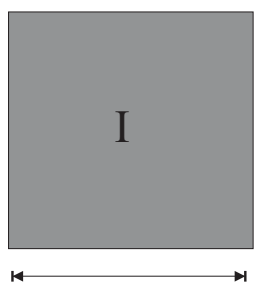

1

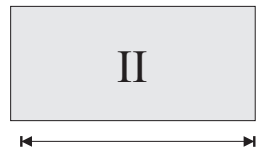

1

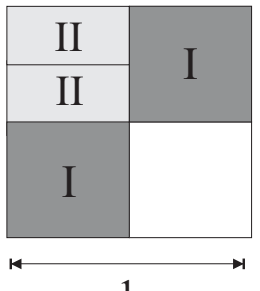

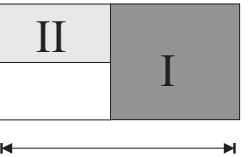

1

Figure 5. An irreducible graph-directed construction. 
Example 9. Figure 6 is a construction of Penrose fractals. Here the dimension of two connected limit sets is $\log 5 / \log \left(\frac{\sqrt{5}+3}{2}\right)$. Then by Theorem 1 for typical parameters, the corresponding section has dimension $\log 5 / \log \left(\frac{\sqrt{5}+3}{2}\right)-1$.

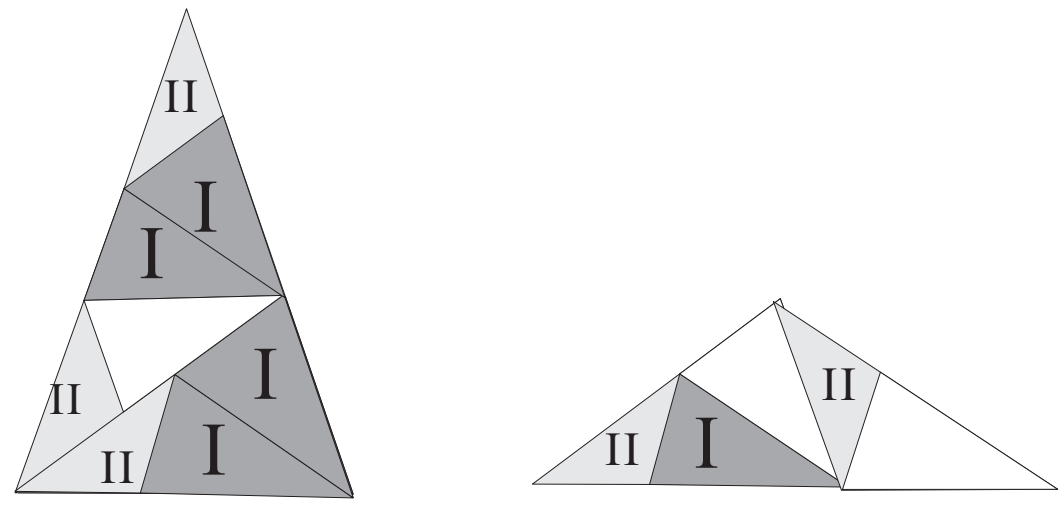

Figure 6. An irreducible construction of Penrose fractals.

1.4. Exceptional cases: Theorems 2 and 3. In Theorem 1, we focus on the typical directions, but what about the exceptional directions? The following is our second result about exceptional directions.

Theorem 2. Suppose $\left\{K_{1}, \cdots, K_{l}\right\}$ are graph-directed sets satisfying (1.1) and (1.2). Assume $\operatorname{dim}_{H} K_{1}=\cdots=\operatorname{dim}_{H} K_{i}=s \geq m$. If $V \in G(n, n-m)$, then there exist constants $c_{1}, c_{2}, c_{3}, c_{4}$ depending on $V$, satisfying $c_{2}, c_{4} \in\left[c_{1}, c_{3}\right]$ and $c_{3} \leq(s-m)$ such that for $\mathscr{H}^{m}$ almost all $a \in P_{V^{\perp}}\left(K_{j}\right)$,

$$
\begin{aligned}
& \operatorname{dim}_{H}\left[(V+a) \cap K_{j}\right]=c_{1}, \underline{\operatorname{dim}}_{B}\left[(V+a) \cap K_{j}\right]=c_{2}, \\
& \overline{\operatorname{dim}}_{B}\left[(V+a) \cap K_{j}\right]=c_{3}, \operatorname{dim}_{P}\left[(V+a) \cap K_{j}\right]=c_{4} .
\end{aligned}
$$

As the self-similar structure is a special irreducible graph-directed construction, we have the following corollary:

Corollary 2. Given similitudes $S_{i}(x)=\rho_{i} R_{i}(x)+b_{i}(1 \leq i \leq k)$, let $E$ denote the self-similar set generated by $\left\{S_{i}\right\}_{i}$. Suppose that the set

$$
\left\{R_{i}\right\}_{i} \text { is contained in a finite subgroup of } O(n) \text {. }
$$

Then assumption (1.2) holds. Given $V \in G(n, n-m)$ for some $j$, then there exist constants $c_{1}, c_{2}, c_{3}, c_{4}$ only depending on $V$ and $E$, satisfying $c_{2}, c_{4} \in\left[c_{1}, c_{3}\right]$ and $c_{3} \leq(s-m)$ such that for $\mathscr{H}^{m}$ almost all $a \in P_{V^{\perp}}(E)$,

$$
\begin{aligned}
& \operatorname{dim}_{H}[(V+a) \cap E]=c_{1}, \underline{\operatorname{dim}}_{B}[(V+a) \cap E]=c_{2}, \\
& \overline{\operatorname{dim}}_{B}[(V+a) \cap E]=c_{3}, \operatorname{dim}_{P}[(V+a) \cap E]=c_{4} .
\end{aligned}
$$

Remark 2. As in Example 4 (or 5), for the fixed direction $\theta=\pi / 4$ and for almost all $a \in J_{\theta}$ (or $\mathscr{J}_{\theta}$ ), the Hausdorff dimension of sections is not $\log 8 / \log 3-1$ (or $\log 4 / \log 3-1$ ). That means the exceptional set is not empty, that is,

$$
\begin{aligned}
& \left\{V: \mathscr{H}^{m}\left\{a \in P_{V^{\perp}}(E): \operatorname{dim}\left(E_{V, a}\right)=s-m\right\}=\mathscr{H}^{m}\left(P_{V^{\perp}}(E)\right)>0\right\} \\
& \varsubsetneqq\left\{V: \mathscr{H}^{m}\left(P_{V^{\perp}}(E)\right)>0\right\} .
\end{aligned}
$$

Remark 3. Theorem 2 is valid for each fixed direction but only meaningful for the direction $V$ satisfying $\mathscr{H}^{m}\left(P_{V^{\perp}}\left(K_{i}\right)\right)>0$ for all $i$. 
Remark 4. In Theorems 1 and 2, similitudes need not satisfy the open set condition of the graph-directed construction. Also, we do not need the condition $0<\mathscr{H}^{s}\left(K_{i}\right)<\infty$ appearing in Marstrand's theorem.

For every self-similar set $E$, we always have $\mathscr{H}^{s}(E)<\infty$ ( e.g., see [Fa3]). But $\mathscr{H}^{s}(E)>0$ maybe fails. For example, when $s=\operatorname{dim}_{H} E$ is the self-similar dimension, that is, $\sum_{i=1}^{k} \rho_{i}^{s}=1$, then by Schief's theorem [S], we notice that $\mathscr{H}^{s}(E)>0$ if and only if the family of similitudes satisfies the open set condition.

Let dim be any dimension function on the subsets of $\mathbf{R}^{n}$ satisfying the following three conditions:

(C1) $\operatorname{dim}(A) \leq \operatorname{dim}(B)$ if $A \subset B \subset \mathbf{R}^{n}$;

(C2) $\operatorname{dim}(A) \leq \operatorname{dim}(S(A))$ for any contracting similitude $S: \mathbf{R}^{n} \rightarrow \mathbf{R}^{n}$ and $A \subset$ $\mathbf{R}^{n}$

(C3) Given a Borel set $A \subset \mathbf{R}^{n}$ and $V \in G(n, n-m)$, the function $f: V^{\perp} \rightarrow \mathbf{R}$ defined by $f(a)=\operatorname{dim}[(V+a) \cap A)]$ is $\mathscr{H}^{m}$-measurable.

For example, $\operatorname{dim}_{H}, \operatorname{dim}_{P}, \underline{\operatorname{dim}}_{B}, \overline{\operatorname{dim}}_{B}$ satisfy these conditions, and $\operatorname{dim}(A)=$ $\operatorname{dim}(S(A))$ here (For (C3), see [MM] and Proposition 2).

We have a generalization of Theorem 2 :

Theorem 3. Suppose $\left\{K_{1}, \cdots, K_{l}\right\}$ are graph-directed sets satisfying (1.1) and (1.2). Assume $\operatorname{dim}_{H} K_{1}=\cdots=\operatorname{dim}_{H} K_{i}=s \geq m$. Let dim be any dimension function satisfying (C1)-(C3). If $V \in G(n, n-m)$, then there exist constants $c$ depending on $V$ and dim such that for all $j$ and for $\mathscr{H}^{m}$-almost all $a \in P_{V^{\perp}}\left(K_{j}\right)$,

$$
\operatorname{dim}\left[(V+a) \cap K_{j}\right]=c .
$$

The rest of the paper is organized as follows. In Section 2, we obtain some preliminary on sections, including a weaker Marstrand's theorem and a typical estimation of the upper Box dimension of plane sections. In Section 3, we provide the structure of the projection for graph-directed sets satisfying assumption (1.2). In particular, the projection of the scaling self-similar set is a scaling self-similar set. In Section 4, we provide a proposition of ergodic type for general graph-directed sets without assumption (1.2). Using the proposition of ergodic type, we prove Theorems in Section 5. In Section 3, Section 4 and Section 5, we deal with scaling self-similar sets before the graph-directed sets, because the method of dealing with the scaling self-similar sets is easy to read and leads to the general method.

\section{Preliminaries on sections}

Recall some classical results:

(1) Projection theorem (e.g., see [Mat5]): If $E \subset \mathbf{R}^{n}$ is a Borel set with $\operatorname{dim}_{H} E>$ $m$, then for $\gamma_{n, m}$-a.e. $V \in G(n, m), \mathscr{H}^{m}\left[P_{V}(E)\right]>0$.

(2) Marstrand's theorem (e.g., see [Mat5]): Suppose $m \leq s \leq n$ and $A \subset \mathbf{R}^{n}$ is a Borel set with $0<\mathscr{H}^{s}(A)<\infty$. Then for $\gamma_{n, n-m}$-a.e. $V \in G(n, n-m)$,

$$
\mathscr{H}^{m}\left\{a \in V^{\perp}: \operatorname{dim}_{H}[A \cap(V+a)]=s-m\right\}>0 .
$$

(3) If $A \subset \mathbf{R}^{n}$ is a Borel set and $0 \leq t<\operatorname{dim}_{H} A$, then there is a compact set $B \subset A$ satisfying $0<\mathscr{H}^{t}(B)<\infty$ (e.g., see [Fa2]). 
Proposition 1. Suppose $m \leq s \leq n$, and $A \subset \mathbf{R}^{n}$ is a Borel set with $\operatorname{dim}_{H} A=$ $s$. Then for any fixed $t$ with $m \leq t<s$, for $\gamma_{n, n-m}$-a.e. $V \in G(n, n-m)$,

$$
\mathscr{H}^{m}\left\{a \in V^{\perp}: \operatorname{dim}_{H}[A \cap(V+a)] \geq t-m\right\}>0 .
$$

Proof. By the above classical result (3), there is a compact set $B \subset A$, such that $0<\mathscr{H}^{t}(B)<\infty$. It follows from Marstrand's theorem that for a.e. $V$,

$$
\begin{aligned}
& \mathscr{H}^{m}\left\{a \in V^{\perp}: \operatorname{dim}_{H}[A \cap(V+a)] \geq t-m\right\} \\
& \geq \mathscr{H}^{m}\left\{a \in V^{\perp}: \operatorname{dim}_{H}[B \cap(V+a)] \geq t-m\right\}>0 .
\end{aligned}
$$

Proposition 2. [MM] The following functions

$$
\begin{aligned}
& f_{1}(a)=\operatorname{dim}_{H}\left[K \cap\left(W^{\perp}+a\right)\right], f_{4}(a)=\operatorname{dim}_{P}\left[K \cap\left(W^{\perp}+a\right)\right], \\
& f_{2}(a)=\underline{\operatorname{dim}}_{B}\left[K \cap\left(W^{\perp}+a\right)\right], f_{3}(a)=\overline{\operatorname{dim}}_{B}\left[K \cap\left(W^{\perp}+a\right)\right],
\end{aligned}
$$

are $\mathscr{H}^{m}$-measurable for any compact set $K$ and any $m$-dimensional subspace $W \subset$ $\mathbf{R}^{n}$.

2.1. Estimation of upper box dimension. The following proposition is an analogue of some classical results (see Lemma 5 of [Fa1] and Chapter 10 of [Mat5]): If $F \subset \mathbf{R}^{n}$ and $V \in G(n, n-m)$,

$$
\begin{aligned}
& \operatorname{dim}_{P}[(V+a) \cap F] \leq \max \left\{0, \operatorname{dim}_{P} F-m\right\}, \\
& \operatorname{dim}_{H}[(V+a) \cap F] \leq \max \left\{0, \operatorname{dim}_{H} F-m\right\},
\end{aligned}
$$

for $\mathscr{H}^{m}$-almost all $a \in V^{\perp}$.

Proposition 3. Given $F \subset \mathbf{R}^{n}$ and an $(n-m)$-dimensional subspace $V$, then for $\mathscr{H}^{m}$-almost all $a \in V^{\perp}$ we have

$$
\overline{\operatorname{dim}}_{B}[(V+a) \cap F] \leq \max \left\{0, \overline{\operatorname{dim}}_{B} F-m\right\} .
$$

The proof is standard, please refer to the proof of Lemma 5 in [Fa1].

\section{Projection of graph-directed set}

Given an $(n-m)$-dimensional subspace $V \subset \mathbf{R}^{n}$, let $P_{V^{\perp}}: \mathbf{R}^{n} \rightarrow V^{\perp}$ be the orthogonal projection from $\mathbf{R}^{n}$ onto $V^{\perp}$.

3.1. Version of scaling self-similar sets. Suppose $F_{i}(x)=\rho_{i} x+b_{i}(1 \leq i \leq$ $k$ ), and $E$ is the self-similar set generated by $\left\{F_{i}\right\}_{i=1}^{k}$, i.e., $E=\bigcup_{i=1}^{k} F_{i}(E)$. Now, a family $\left\{S_{i}\right\}_{i=1}^{k}$ of the self-contractions of $V^{\perp}$ is defined by

$$
S_{i}(x)=\left.P_{V^{\perp}} \circ F_{i}\right|_{V^{\perp}}=\rho_{i} x+P_{V^{\perp}}\left(b_{i}\right), \forall x \in V^{\perp}(1 \leq i \leq k) .
$$

We have the following proposition.

Proposition 4. $J_{V}=P_{V^{\perp}}(E) \subset V^{\perp}$ is a self-similar set, that is,

$$
J_{V}=\bigcup_{i} S_{i}\left(J_{V}\right)
$$

Proof. In fact, we have

$$
P_{V^{\perp}}(E)=\bigcup_{i=1}^{k}\left(P_{V^{\perp}} \circ F_{i}\right)(E)=\bigcup_{i=1}^{k}\left(\left.P_{V^{\perp}} \circ F_{i}\right|_{V^{\perp}} \circ P_{V^{\perp}}\right) E=\bigcup_{i=1}^{k} S_{i}\left(P_{V^{\perp}} E\right),
$$

and thus, $J_{V}=P_{V^{\perp}}(E)$ is the invariant set of $\left\{S_{i}\right\}_{i=1}^{k}$. 
In general, we may consider some dimension function dim satisfying (C1) and (C2) as follows.

(C1) $\operatorname{dim}(A) \leq \operatorname{dim}(B)$ if $A \subset B \subset \mathbf{R}^{n}$;

(C2) $\operatorname{dim}(A) \leq \operatorname{dim}(S(A))$ for any contracting similitude $S: \mathbf{R}^{n} \rightarrow \mathbf{R}^{n}$ and $A \subset$ $\mathbf{R}^{n}$.

For example, $\operatorname{dim}_{H}, \operatorname{dim}_{P}, \underline{\operatorname{dim}}_{B}, \overline{\operatorname{dim}}_{B}$ satisfy these conditions with $\operatorname{dim}(A)=$ $\operatorname{dim}(S(A))$.

Now, we have the following proposition.

Proposition 5. For any dim satisfying $(\mathrm{C} 1)$ and $(\mathrm{C} 2)$,

$$
\operatorname{dim} E_{V, S_{i}(a)} \geq \operatorname{dim} E_{V, a}
$$

Proof. In fact,

$$
\begin{aligned}
E_{V, a} & =(V+a) \cap E=\bigcup_{i=1}^{k}\left[(V+a) \cap F_{i}(E)\right] \\
& =\bigcup_{i=1}^{k} F_{i} F_{i}^{-1}\left[(V+a) \cap F_{i}(E)\right]=\bigcup_{i=1}^{k} F_{i}\left[F_{i}^{-1}(V+a) \cap(E)\right],
\end{aligned}
$$

with

$$
\begin{aligned}
F_{i}^{-1}(V+a) & =\rho_{i}^{-1}(V+a)-\rho_{i}^{-1} b_{i} \quad\left(\operatorname{asb}_{i}=P_{V} b_{i}+P_{V^{\perp}} b_{i}\right) \\
& =\left[\rho_{i}^{-1} V-\rho_{i}^{-1}\left(P_{V} b_{i}\right)\right]+\left[\rho_{i}^{-1} a-\rho_{i}^{-1} P_{V^{\perp}} b_{i}\right]=V+S_{i}^{-1}(a),
\end{aligned}
$$

and thus

$$
E_{V, a}=\bigcup_{i=1}^{k} F_{i}\left[\left(V+S_{i}^{-1}(a)\right) \cap(E)\right]=\bigcup_{i=1}^{k} F_{i}\left(E_{V, S_{i}^{-1}(a)}\right)=\bigcup_{i, S_{i}^{-1}(a) \in J_{V}} F_{i}\left(E_{V, S_{i}^{-1}(a)}\right),
$$

where $F_{i}\left(E_{V, S_{i}^{-1}(a)}\right)$ is a similar copy of $E_{V, S_{i}^{-1}(a)}$. Then it follows from $(\mathrm{C} 1)$ and $(\mathrm{C} 2)$ that

and thus

$$
\operatorname{dim}\left[F_{i}\left(E_{V, S_{i}^{-1}(a)}\right)\right] \geq \operatorname{dim} E_{V, S_{i}^{-1}(a)}
$$

$$
\operatorname{dim} E_{V, a} \geq \max _{i, S_{i}^{-1}(a) \in J_{V}} \operatorname{dim} E_{V, S_{i}^{-1}(a)} .
$$

In particular, as $a=S_{i}^{-1}\left(S_{i} a\right) \in J_{V}$,

$$
\operatorname{dim} E_{V, S_{i}(a)} \geq \operatorname{dim} E_{V, a} .
$$

3.2. Version of graph-directed constructions. For each directed edge $e$ in the irreducible graph $G$, we have a similitude

$$
S_{e}(x)=\rho_{e} R_{e} x+b_{e} .
$$

Then

$$
A_{i, j}=\left\{R_{e_{1}} \circ \cdots \circ R_{e_{k}}: e_{1} \cdots e_{k} \text { is a path from } i \text { to } j\right\} .
$$

From the irreducibility of the graph, $A_{i, j}$ is non-empty for any $1 \leq i, j \leq l$.

Given $W \in G(n, m)$, let $P_{W}: \mathbf{R}^{n} \rightarrow W$ be the orthogonal projection from $\mathbf{R}^{n}$ onto $W$. Notice that for any orthogonal transformation $R$,

$$
R^{-1} P_{W} R=P_{R^{-1} W}
$$


Since $K_{i}=\bigcup_{j} \bigcup_{e \in \Gamma_{i, j}} S_{e}\left(K_{i}\right)$, we have

$$
\begin{aligned}
\left(P_{W} K_{i}\right) & =\bigcup_{j} \bigcup_{e \in \Gamma_{i, j}} P_{W} S_{e}\left(K_{j}\right)=\bigcup_{j} \bigcup_{e \in \Gamma_{i, j}}\left[\rho_{e} P_{W} R_{e}\left(K_{j}\right)+P_{W} b_{e}\right] \\
& =\bigcup_{j} \bigcup_{e \in \Gamma_{i, j}}\left[\rho_{e} R_{e}\left(R_{e}^{-1} P_{W} R_{e}\right)\left(K_{j}\right)+P_{W} b_{e}\right] .
\end{aligned}
$$

Then (3.3) yields

$$
\left(P_{W} K_{i}\right)=\bigcup_{j} \bigcup_{e \in \Gamma_{i, j}}\left[\rho_{e} R_{e}\left(P_{R_{e}^{-1} W}\left(K_{j}\right)\right)+P_{W} b_{e}\right] .
$$

That means that $\left(P_{W} K_{i}\right)$ includes a similar copy of $P_{R_{e}^{-1} W}\left(K_{j}\right)$, where $e$ is an edge from $i$ to $j$ and the similitude from $\left(P_{R_{e}^{-1} W}\left(K_{j}\right) \subset\right) R_{e}^{-1} W$ to $\left(P_{W}\left(K_{i}\right) \subset\right) W$ is

$$
S(x)=\left.\rho_{e} R_{e}\right|_{R_{e}^{-1} W}(x)+P_{W} b_{e} .
$$

Fix $W^{*} \in G(n, m)$ and $1 \leq j^{*} \leq l$, and define

$$
\Xi\left(W^{*}, j^{*}\right)=\bigcup_{i}\left\{\left(R^{-1} W^{*}, i\right): R \in A_{j^{*}, i}\right\} .
$$

Then there is a graph-directed construction on the graph with the vertex set $\Xi\left(W^{*}, j^{*}\right)$.

(1) For each $(W, i) \in \Xi\left(W^{*}, j^{*}\right)$, we have the compact set

$$
K_{(W, i)}=P_{W} K_{i} .
$$

(2) For any $(W, i),\left(W^{\prime}, i^{\prime}\right) \in \Xi\left(W^{*}, j^{*}\right)$, if there is an edge $e$ from $i$ to $i^{\prime}$ in the graph $G$ such that

$$
W^{\prime}=R_{e}^{-1} W,
$$

then we consider the edge $e$ as an edge in $\Xi\left(W^{*}, j^{*}\right)$ from $(W, i)$ to $\left(W^{\prime}, i^{\prime}\right)$, which is still denoted by $e$. For this edge, let

$$
T_{e}(x)=\left.\rho_{e} R_{e}\right|_{W^{\prime}}(x)+P_{W} b_{e}
$$

be the contracting similitude from $\left(P_{W^{\prime}} K_{i^{\prime}} \subset\right) W^{\prime}$ to $\left(P_{W} K_{i} \subset\right) W$ with respect to $e$ such that $T_{e}(x)\left(P_{W^{\prime}} K_{i^{\prime}}\right) \subset P_{W} K_{i}$.

Let $\Gamma_{(W, i),\left(W^{\prime}, i^{\prime}\right)}$ be the set of all the edges from $(W, i)$ to $\left(W^{\prime}, i^{\prime}\right)$ and $\Gamma_{(W, i),\left(W^{\prime}, i^{\prime}\right)}^{k}$ the set of all the paths from $(W, i)$ to $\left(W^{\prime}, i^{\prime}\right)$ of length $k$.

(3) By (3.4), we have

$$
K_{(W, i)}=\bigcup_{\left(W^{\prime}, i^{\prime}\right)} \bigcup_{e \in \Gamma(W, i),\left(W^{\prime}, i^{\prime}\right)} T_{e}\left[K_{\left(W^{\prime}, i^{\prime}\right)}\right] .
$$

Naturally, for each positive integer $k$,

$$
K_{(W, i)}=\bigcup_{\left(W^{\prime}, i^{\prime}\right)} \bigcup_{e^{*} \in \Gamma_{(W, i),\left(W^{\prime}, i^{\prime}\right)}^{k}} T_{e^{*}}\left[K_{\left(W^{\prime}, i^{\prime}\right)}\right],
$$

where $T_{e^{*}}=T_{e_{1}} \circ \cdots \circ T_{e_{k}}$ for $e=e_{1} \cdots e_{k}$.

Now, in the above construction, there is an edge $e$ in $G$ from $i$ to $j$, if and only if there is an edge from $(W, i)$ to $\left(R_{e}^{-1} W, j\right)$. An important fact is that the above construction is irreducible. 
Proposition 6. $\Xi\left(W^{*}, j^{*}\right)=\bigcup_{i} \bigcup_{R \in A_{j^{*}, i}}\left(R^{-1} W^{*} \times\{i\}\right)$ is irreducible under the above construction.

Proof. In fact, for any $(W, i),\left(W^{\prime}, i^{\prime}\right) \in \Xi\left(W^{*}, j^{*}\right)$, one has

$$
\begin{aligned}
W & =R^{-1} W^{*} \text { with } R \in A_{j^{*}, i}, \\
W^{\prime} & =\left(R^{\prime}\right)^{-1} W^{*} \text { with } R^{\prime} \in A_{j^{*}, i^{\prime}} .
\end{aligned}
$$

Now we seek for a path from $(W, i)$ to $\left(W^{\prime}, i^{\prime}\right)$.

By (2) of Lemma 1, $A_{j^{*}, i^{\prime}}=A_{j^{*}, i} A_{i, i^{\prime}}$, so there is a path $e^{*}=e_{1} \cdots e_{k}$ from $i$ to $i^{\prime}$, passing through $i=j_{0}, j_{1}, \cdots, j_{k}=i^{\prime}$, such that $R^{\prime}=R \cdot R_{e^{*}}$, which implies

$$
W^{\prime}=\left(R^{\prime}\right)^{-1} W^{*}=R_{e_{k}}^{-1} \cdots R_{e_{1}}^{-1} R^{-1} W^{*}
$$

Therefore, there is a path from $(W, i)$ to $\left(W^{\prime}, i^{\prime}\right)$ :

$$
\begin{aligned}
(W, i) & =\left(R^{-1} W^{*}, j_{0}\right) \\
& \rightarrow\left(R_{e_{1}}^{-1} R^{-1} W^{*}, j_{1}\right) \in A_{j^{*}, j_{1}}^{-1} \times\left\{j_{1}\right\} \subset \Xi \\
& \rightarrow \cdots \\
& \rightarrow\left(W^{\prime}, i^{\prime}\right)=\left(R_{e_{k}}^{-1} \cdots R_{e_{1}}^{-1} R^{-1} W^{*}, j_{k}\right) \in A_{j^{*}, j_{k}}^{-1} \times\left\{j_{k}\right\} \subset \Xi .
\end{aligned}
$$

Example 10. For the Koch curve, $\left\{R_{e}\right\}_{e}=\left\{1,1, e^{i \pi / 3}, e^{-i \pi / 3}\right\}$. Given $W \in$ $G(2,1)$, let $W_{k}=e^{i k \pi / 3} W$ for $0 \leq k \leq 5$. Here Figure 7 is the graph for $\Xi=\Xi(W, 1)$.

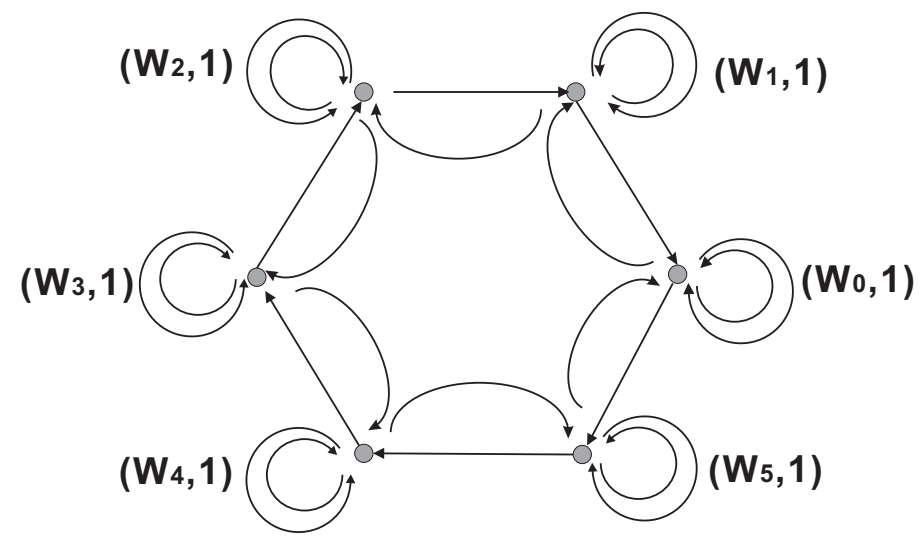

Figure 7. $\Xi=\Xi(W, 1)$ for the Koch curve.

Given $W^{*} \in G(n, m)$ and $j^{*} \in \mathbf{N} \cap[1, l]$, let

$$
\Xi=\bigcup_{i} A_{j^{*}, i}^{-1} W^{*} \times\{i\}
$$

For $(W, i) \in \Xi$, a function $g_{(W, i)}: W \rightarrow \mathbf{R}$ is defined by

$$
g_{(W, i)}(x)=\operatorname{dim}\left[\left(W^{\perp}+x\right) \cap K_{i}\right] \text { for } x \in W,
$$

where dim is any dimension function satisfying (C1) and (C2).

We need the following proposition:

Proposition 7 . For any $\left(W_{1}, i_{1}\right),\left(W_{2}, i_{2}\right) \in \Xi$ and any edge $e$ from $\left(W_{1}, i_{1}\right)$ to $\left(W_{2}, i_{2}\right)$,

$$
g_{\left(W_{1}, i_{1}\right)}\left(T_{e}(x)\right) \geq g_{\left(W_{2}, i_{2}\right)}(x)
$$


that is

$$
\operatorname{dim}\left[\left(W_{1}^{\perp}+T_{e}(x)\right) \cap K_{i_{1}}\right] \geq \operatorname{dim}\left[\left(W_{2}^{\perp}+x\right) \cap K_{i_{2}}\right]
$$

for any $x \in P_{W_{2}} K_{i_{2}}$. Here (C1) and (C2) hold.

Proof. Here $W_{2}=R_{e}^{-1} W_{1}, W_{2}^{\perp}=R_{e}^{-1} W_{1}^{\perp}$ with the edge $e$ from $i_{1}$ to $i_{2}$ in the graph $G$, and for $x \in W_{2}$,

$$
T_{e}(x)=\left.\rho_{e} R_{e}\right|_{W_{2}}(x)+P_{W_{1}} b_{e} .
$$

It follows from the graph-directed construction that

$$
K_{i_{1}} \supset S_{e}\left(K_{i_{2}}\right)
$$

where $S_{e}(x)=\rho_{e} R_{e}(x)+b_{e}$. Hence,

$$
\begin{aligned}
\left(W_{1}^{\perp}+T_{e}(x)\right) \cap K_{i_{1}} & \supset\left(W_{1}^{\perp}+T_{e}(x)\right) \cap\left(S_{e}\left(K_{i_{2}}\right)\right) \\
& =S_{e} S_{e}^{-1}\left[\left(W_{1}^{\perp}+T_{e}(x)\right) \cap\left(S_{e}\left(K_{i_{2}}\right)\right)\right] \\
& =S_{e}\left[\left(S_{e}^{-1}\left(W_{1}^{\perp}+T_{e}(x)\right)\right) \cap K_{i_{2}}\right] .
\end{aligned}
$$

As $S_{e}$ is a similitude and (C1), (C2) hold, we have

$$
\begin{aligned}
\operatorname{dim}\left[\left(W_{1}^{\perp}+T_{e}(x)\right) \cap K_{i_{1}}\right] & \geq \operatorname{dim}\left\{S_{e}\left[\left(S_{e}^{-1}\left(W_{1}^{\perp}+T_{e}(x)\right)\right) \cap K_{i_{2}}\right]\right\} \\
& \geq \operatorname{dim}\left[\left(S_{e}^{-1}\left(W_{1}^{\perp}+T_{e}(x)\right)\right) \cap K_{i_{2}}\right],
\end{aligned}
$$

where

$$
\begin{aligned}
\left.S_{e}^{-1}\left(W_{1}^{\perp}+T_{e}(x)\right)\right) & =\rho_{e}^{-1} R_{e}^{-1}\left[W_{1}^{\perp}+\left(T_{e} x-b_{e}\right)\right] \\
& =W_{2}^{\perp}+\rho_{e}^{-1} R_{e}^{-1}\left[\rho_{e} R_{e}(x)+P_{W_{1}} b_{e}-b_{e}\right] \\
& =W_{2}^{\perp}+x+\rho_{e}^{-1} R_{e}^{-1}\left(P_{W_{1}^{\perp}} b_{i}\right)=W_{2}^{\perp}+x,
\end{aligned}
$$

since

Therefore,

$$
\rho_{e}^{-1}\left[R_{e}^{-1}\left(P_{W_{1}^{\perp}} b_{i}\right)\right] \in \rho_{e}^{-1}\left[R_{e}^{-1} W_{1}^{\perp}\right]=\rho_{e}^{-1} W_{2}^{\perp}=W_{2}^{\perp} .
$$

$$
\operatorname{dim}\left[\left(W_{1}^{\perp}+T_{e}(x)\right) \cap K_{i_{1}}\right] \geq \operatorname{dim}\left[\left(W_{2}^{\perp}+x\right) \cap K_{i_{2}}\right]
$$

\section{Result of ergodic type}

4.1. Version of scaling self-similar sets. Suppose that $\left\{T_{i}(x)=\rho_{i} R_{i}(x)+\right.$ $\left.b_{i}\right\}_{i=1}^{k}$ is a family of contracting similitudes of $\mathbf{R}^{m}$, where $\left\{R_{i}\right\}_{i=1}^{k}$ are orthogonal transformations of $\mathbf{R}^{m}$. Let $F\left(\subset \mathbf{R}^{m}\right)$ denote the self-similar set generated by $\left\{T_{i}\right\}_{i=1}^{k}$. For notational convenience, write

$$
T_{i_{1} \cdots i_{k}}=T_{i_{1}} \circ \cdots \circ T_{i_{k}} .
$$

Proposition 8. If $B \subset F$ is an $\mathscr{H}^{m}$-measurable set such that

$$
\cup_{i=1}^{k} T_{i}(B) \subset B,
$$

then $\mathscr{H}^{m}(B)=\mathscr{H}^{m}(F)$ or 0 .

Proof. Without loss of generality, we suppose $\mathscr{H}^{m}(F)>0$. On the contrary, we may assume $0<\mathscr{H}^{m}(B)<\mathscr{H}^{m}(F)$. Then

$$
0<\mathscr{H}^{m}(F \backslash B)<\mathscr{H}^{m}(F) .
$$

Since $\cup_{i} T_{i}(B) \subset B$, we have $T_{i_{1} \cdots i_{p}}(B) \subset B$. 
Obviously, we have

$$
\left(T_{i_{1} \cdots i_{p}}\right)^{-1}(F \backslash B) \subset \mathbf{R}^{m} \backslash B \text { for any } i_{1} \cdots i_{p} .
$$

Since $\mathscr{H}^{m}(F \backslash B)>0$, we can take a Lebesgue point $x_{0} \in F \backslash B$ with $\mathscr{H}^{m}$-density 1, which implies that for any $\delta>0$ there exists $\varepsilon_{0}>0$ such that

$$
\frac{\mathscr{H}^{m}[I \cap(F \backslash B)]}{\mathscr{H}^{m}(I)} \geq 1-\delta,
$$

whenever $x_{0}$ is the center of the ball $I \subset \mathbf{R}^{m}$ of diameter diam $(I) \leq \varepsilon_{0}$.

Take an integer $p$ such that $2\left(\max _{i} \rho_{i}\right)^{p} \operatorname{diam}(F) \leq \varepsilon_{0}$. As $F=\cup_{i} T_{i}(F)$,

$$
F=\bigcup_{j_{1} \cdots j_{p}} T_{j_{1} \cdots j_{p}}(F) .
$$

Since $x_{0} \in F$, we may assume $x_{0} \in T_{i_{1} \cdots i_{p}}(F)$ for a certain sequence $i_{1} \cdots i_{p} \in$ $\{1, \cdots, k\}^{p}$. Then let $y=\left(T_{i_{1} \cdots i_{p}}\right)^{-1}\left(x_{0}\right) \in F$. Choose a minimal ball $I^{*}$ centered at $y$ and covering $F$, which implies $F \subset I^{*}$, and the diameter of $I^{*}$ is less than $2 \operatorname{diam}(F)$. Then the ball $I=T_{i_{1} \cdots i_{p}}\left(I^{*}\right)$ centered at $x_{0}$ with diameter

$$
\operatorname{diam}(I)=\left(\rho_{i_{1}} \cdots \rho_{i_{p}}\right) \operatorname{diam}\left(I^{*}\right) \leq\left(\max _{i} \rho_{i}\right)^{p} \operatorname{diam}\left(I^{*}\right) \leq 2\left(\max _{i} \rho_{i}\right)^{p} \operatorname{diam}(F) \leq \varepsilon_{0} .
$$

Therefore, by (4.2), we have

$$
\begin{aligned}
\frac{\mathscr{H}^{m}\left[\left(T_{i_{1} \cdots i_{p}}\right)^{-1}(I \cap(F \backslash B))\right]}{\mathscr{H}^{m}\left[\left(T_{i_{1} \cdots i_{p}}\right)^{-1}(I)\right]} & =\frac{\left(\rho_{i_{1}} \cdots \rho_{i_{p}}\right)^{-m} \mathscr{H}^{m}[I \cap(F \backslash B)]}{\left(\rho_{i_{1}} \cdots \rho_{i_{p}}\right)^{-m} \mathscr{H}^{m}(I)} \\
& \geq \frac{\mathscr{H}^{m}[I \cap(F \backslash B)]}{\mathscr{H}^{m}(I)} \geq 1-\delta .
\end{aligned}
$$

We also have

$$
\begin{aligned}
\left(T_{i_{1} \cdots i_{p}}\right)^{-1}[I \cap(F \backslash B)] & \subset\left(T_{i_{1} \cdots i_{p}}\right)^{-1}(F \backslash B) \cap\left(T_{i_{1} \cdots i_{p}}\right)^{-1}(I) \\
& \subset\left(\mathbf{R}^{m} \backslash B\right) \cap I^{*}=I^{*} \backslash B
\end{aligned}
$$

and $\left(T_{i_{1} \cdots i_{p}}\right)^{-1}(I)=I^{*}$. This implies that

$$
\frac{\mathscr{H}^{m}\left(I^{*} \backslash B\right)}{\mathscr{H}^{m}\left(I^{*}\right)} \geq \frac{\mathscr{H}^{m}\left[\left(T_{i_{1} \cdots i_{p}}\right)^{-1}[I \cap(F \backslash B)]\right]}{\mathscr{H}^{m}\left[\left(T_{i_{1} \cdots i_{p}}\right)^{-1}(I)\right]} \geq 1-\delta .
$$

On the other hand, since the radius of $I^{*}$ is less than $\operatorname{diam}(F)$, by [Mat5],

$$
\mathscr{H}^{m}\left(I^{*}\right)=2^{m} \alpha(m)^{-1} \mathscr{L}^{m}\left(I^{*}\right) \leq 2^{m}[\operatorname{diam}(F)]^{m},
$$

we have

$$
\frac{\mathscr{H}^{m}\left(I^{*} \backslash B\right)}{\mathscr{H}^{m}\left(I^{*}\right)}=1-\frac{\mathscr{H}^{m}(B)}{\mathscr{H}^{m}\left(I^{*}\right)} \leq 1-\frac{\mathscr{H}^{m}(B)}{2^{m}[\operatorname{diam}(F)]^{m}} .
$$

This is in contradiction with the inequality (4.3) when $\delta$ is small enough so that $\delta<\frac{\mathscr{H}^{m}(B)}{2^{m}[\operatorname{diam}(F)]^{m}}$.

Remark 5. The condition $\bigcup_{i=1}^{k} T_{i}(B) \subset B$ is like that of the definition of the upper self-similar set [Fa3]. However, here we only need the assumption that $B$ is a Lebesgue measurable set. 
Corollary 3. Suppose $f: F \rightarrow \mathbf{R}$ is an $\mathscr{H}^{m}$-measurable function such that for any $a \in F$ and $1 \leq i \leq k$,

$$
f\left(T_{i}(a)\right) \geq f(a)
$$

Then

$$
f(a)=d
$$

for $\mathscr{H}^{m}$-almost all $a \in F$.

Proof. Assume $\mathscr{H}^{m}(F)>0$. Let $d$ be the $\mathscr{H}^{m}$-essential upper bound of $f$. For any integer $p>0$, let us define the set

$$
C_{p}=\{a \in F: f(a) \geq d-1 / p\} .
$$

It follows from the definition of the essential upper bound that for any $p$,

$$
\mathscr{H}^{m}\left(C_{p}\right)>0 .
$$

As $f\left(T_{i}(a)\right) \geq f(a)$ for any $a \in F$, we have

$$
\bigcup_{i} T_{i}\left(C_{p}\right) \subset C_{p} \text { and } \mathscr{H}^{m}\left(C_{p}\right)>0 \text {. }
$$

Due to Proposition 8, $\mathscr{H}^{m}\left(C_{p}\right)=\mathscr{H}^{m}(F)$.

Consequently, the following subset of $F$

$$
C=\{a \in F: f(a) \geq d\}=\bigcap_{p \geq 1} C_{p}
$$

has full measure $\mathscr{H}^{m}(F)$. Since $d$ is the essential upper bound of $f$, we have

$$
f(a)=d
$$

for $\mathscr{H}^{m}$-almost all $a \in F$.

4.2. Version of graph-directed constructions. We shall obtain a result of ergodic type, in the sense that sets satisfying certain conditions have full measure or measure zero.

Let $\mathfrak{G}$ be an irreducible directed graph including $l$ vertexes $\{1, \cdots, l\}$. For each $i$, there is an $m$-dimensional linear space $V_{i}$ equipped with Euclidean metric and Hausdorff measure $\mathscr{H}^{m}$. For any edge $e$ from $i$ to $j$, there is a contracting similitude $T_{e}: V_{j} \rightarrow V_{i}$. That means

$$
d_{V_{i}}\left(T_{e}(x), T_{e}\left(x^{\prime}\right)\right)=\rho_{e} d_{V_{j}}\left(x, x^{\prime}\right)
$$

for some ratio $\rho_{e} \in(0,1)$.

By [MW], there exists a unique family of compact sets $\left\{M_{1}, \cdots, M_{l}\right\}$ satisfying $M_{i} \subset V_{i}$ and

$$
M_{i}=\bigcup_{j} \bigcup_{e \in \mathscr{E}_{i, j}} T_{e}\left(M_{j}\right)
$$

where $\mathscr{E}_{i, j}$ is the set of all the edges from $i$ to $j$. Let $\mathscr{E}_{i, j}^{k}$ be the set of all the paths of length $k$ from $i$ to $j$. For the path $e_{1} \cdots e_{k}$, let $T_{e^{*}}=T_{e_{1}} \circ \cdots \circ T_{e_{k}}$ and $\rho_{e^{*}}=\rho_{e_{1}} \cdots \rho_{e_{k}}$.

Proposition 9. Suppose $\left\{B_{i}\right\}_{i=1}^{l}$ are $\mathscr{H}^{m}$-measurable sets satisfying

(1) $B_{i} \subset M_{i}$,

(2) $\bigcup_{j} \bigcup_{e \in \mathscr{E}_{i, j}} T_{e}\left(B_{j}\right) \subset B_{i}$. 
Then either

or

$$
\mathscr{H}^{m}\left(B_{i}\right)=\mathscr{H}^{m}\left(M_{i}\right) \text { for all } i,
$$

$$
\mathscr{H}^{m}\left(B_{i}\right)=0 \text { for all } i .
$$

Proof. Without loss of generality, we suppose that there exists $j_{0}$ such that

$$
\mathscr{H}^{m}\left(B_{j_{0}}\right)>0 .
$$

Due to the irreducibility of $\mathfrak{G}$ and the fact that

$$
M_{i}=\bigcup_{j} \bigcup_{e \in \mathscr{E}_{i, j}^{k}} T_{e^{*}}\left(M_{j}\right) \text { for every } k,
$$

for any $i$, the set $B_{i}$ contains $T_{e^{*}}\left(B_{j_{0}}\right)$ for a certain path $e^{*}$, where $T_{e^{*}}\left(B_{j_{0}}\right)$ is a similar copy of $B_{j_{0}}$ and thus has positive $\mathscr{H}^{m}$-measure. Hence,

$$
\mathscr{H}^{m}\left(B_{i}\right)>0 \text { for all } i \text {. }
$$

This also shows that

$$
\mathscr{H}^{m}\left(M_{i}\right)>0 \text { for all } i .
$$

As a result, $\operatorname{diam}\left(M_{i}\right)>0$ for all $i$.

To prove the proposition, we assume on the contrary that $0<\mathscr{H}^{m}\left(B_{i_{0}}\right)<$ $\mathscr{H}^{m}\left(M_{i_{0}}\right)$ for some $i_{0}$. Then

$$
0<\mathscr{H}^{m}\left[M_{i_{0}} \backslash B_{i_{0}}\right]<\mathscr{H}^{m}\left(M_{i_{0}}\right) .
$$

For any path $e^{*}$ passing from $i$ to $j$, we conclude that

$$
\left(T_{e^{*}}\right)^{-1}\left[M_{i} \backslash B_{i}\right] \subset V_{j} \backslash B_{j} .
$$

Otherwise, take a point $b \in\left[\left(T_{e^{*}}\right)^{-1}\left(M_{i} \backslash B_{i}\right)\right] \cap B_{j}$. Then we have

$$
T_{e^{*}}(b) \in M_{i} \backslash B_{i} \text { and } T_{e^{*}}(b) \in T_{e^{*}}\left(B_{j}\right) \subset B_{i},
$$

which yields a contradiction.

Because $\mathscr{H}^{m}\left[M_{i_{0}} \backslash B_{i_{0}}\right]>0$, we can take a Lebesgue point $x_{0} \in M_{i_{0}} \backslash B_{i_{0}}$ with $\mathscr{H}^{m}$-density 1, which implies that for any $\varepsilon>0$, there exists $\delta>0$ such that

$$
\frac{\mathscr{H}^{m}\left[I^{\prime} \cap\left(M_{i_{0}} \backslash B_{i_{0}}\right)\right]}{\mathscr{H}^{m}\left(I^{\prime}\right)} \geq 1-\varepsilon
$$

whenever $I^{\prime} \subset V_{i_{0}}$ is a ball centered at $x_{0}$ with diameter $\operatorname{diam}\left(I^{\prime}\right) \leq \delta$.

Take an integer $p$ such that

$$
2\left(\max _{e} \rho_{e}\right)^{p}\left(\max _{i} \operatorname{diam}\left(M_{i}\right)\right) \leq \delta / 2 .
$$

Then we have

$$
M_{i_{0}}=\bigcup_{j} \bigcup_{e^{*} \in \mathscr{E}_{i_{0}, j}^{p}} T_{e^{*}}\left(M_{j}\right) .
$$

As $x_{0} \in M_{i_{0}}$, we may assume $x_{0} \in T_{e^{*}}\left(M_{j}\right)$ for a certain path $e^{*} \in \mathscr{E}_{i_{0}, j}^{p}$. Let

$$
y=\left(T_{e^{*}}\right)^{-1}\left(x_{0}\right) \in M_{j} \text {. }
$$

Choose a minimal ball $I \subset V_{j}$ centered at $y$ and covering $M_{j}$, which implies $M_{j} \subset I$ and $\operatorname{diam}(I) \leq 2 \operatorname{diam}\left(M_{j}\right)$. Then the ball $I^{*}=T_{e^{*}}(I) \subset V_{i_{0}}$ centered at $x_{0}$ with diameter

$$
\operatorname{diam}\left(I^{*}\right)=\left(\rho_{e^{*}}\right) \operatorname{diam}(I) \leq\left(\max _{e} \rho_{e}\right)^{p} \operatorname{diam}(I) \leq 2\left(\max _{e} \rho_{e}\right)^{p} \operatorname{diam}\left(M_{j}\right) \leq \delta / 2 .
$$


Therefore, by (4.7), we have

$$
\begin{aligned}
\frac{\mathscr{H}^{m}\left[\left(T_{e^{*}}\right)^{-1}\left(I^{*} \cap\left(M_{i_{0}} \backslash B_{i_{0}}\right)\right]\right.}{\mathscr{H}^{m}\left[\left(T_{e^{*}}\right)^{-1}\left(I^{*}\right)\right]} & =\frac{\left(\rho_{e^{*}}\right)^{-m} \mathscr{H}^{m}\left[I^{*} \cap\left(M_{i_{0}} \backslash B_{i_{0}}\right)\right]}{\left(\rho_{e^{*}}\right)^{-m} \mathscr{H}^{m}\left(I^{*}\right)} \\
& =\frac{\mathscr{H}^{m}\left[I^{*} \cap\left(M_{i_{0}} \backslash B_{i_{0}}\right)\right]}{\mathscr{H}^{m}\left(I^{*}\right)} \geq 1-\varepsilon .
\end{aligned}
$$

In fact, since

$$
\left(T_{e^{*}}\right)^{-1}\left[I^{*} \cap\left(M_{i_{0}} \backslash B_{i_{0}}\right)\right]=\left(T_{e^{*}}\right)^{-1}\left(I^{*}\right) \cap\left(T_{e^{*}}\right)^{-1}\left[M_{i_{0}} \backslash B_{i_{0}}\right] \subset I \cap\left(\left(V_{j}\right) \backslash B_{j}\right) \subset I \backslash B_{j},
$$
and $\left(T_{e^{*}}\right)^{-1}\left(I^{*}\right)=I$, we have

$$
\frac{\mathscr{H}^{m}\left(I \backslash B_{j}\right)}{\mathscr{H}^{m}(I)} \geq \frac{\mathscr{H}^{m}\left\{\left(T_{e^{*}}\right)^{-1}\left[I^{*} \cap\left(M_{i_{0}} \backslash B_{i_{0}}\right)\right]\right\}}{\mathscr{H}^{m}\left[\left(T_{e^{*}}\right)^{-1}\left(I^{*}\right)\right]} \geq 1-\varepsilon,
$$

that is,

$$
\frac{\mathscr{H}^{m}\left(I \backslash B_{j}\right)}{\mathscr{H}^{m}(I)} \geq 1-\varepsilon .
$$

On the other hand, $\mathscr{H}^{m}(I)=2^{m} \alpha(m)^{-1} \mathscr{L}^{m}(I) \leq 2^{m}\left[\operatorname{diam}\left(M_{j}\right)\right]^{m}$, and thus

$$
\frac{\mathscr{H}^{m}\left(I \backslash B_{j}\right)}{\mathscr{H}^{m}(I)}=1-\frac{\mathscr{H}^{m}\left(B_{j}\right)}{\mathscr{H}^{m}(I)} \leq 1-\frac{\mathscr{H}^{m}\left(B_{j}\right)}{2^{m}\left[\operatorname{diam}\left(M_{j}\right)\right]^{m}} .
$$

This is in contradiction with the inequality (4.8) when $\varepsilon$ is small enough, so that

$$
\varepsilon<\min _{j^{\prime}} \frac{\mathscr{H}^{m}\left(B_{j^{\prime}}\right)}{2^{m}\left[\operatorname{diam}\left(M_{j^{\prime}}\right)\right]^{m}},
$$

because here $\mathscr{H}^{m}\left(B_{j^{\prime}}\right)>0$ for all $j^{\prime}$ shown above.

Corollary 4. Suppose $g_{i}: M_{i} \rightarrow \mathbf{R}$ is an $\mathscr{H}^{m}$-measurable function for each $1 \leq i \leq l$. If for any edge $e$ and any $x \in M_{j}$,

$$
g_{i}\left(T_{e}(x)\right) \geq g_{j}(x),
$$

then there is a constant $d$ such that for any $i$,

$$
g_{i}(y)=d,
$$

for $\mathscr{H}^{m}$-almost all $y \in M_{i}$.

Proof. Without loss of generality, suppose $\mathscr{H}^{m}\left(M_{i}\right)>0$ for all $i$. Let

$$
d=\max _{i}\left[\operatorname{ess} \sup \left(g_{i}\right)\right] \text {. }
$$

In particular, take $i_{0}$ such that $d=\operatorname{ess} \sup g_{i_{0}}$. For any integer $p>0$, let the set

$$
B_{p, i}=\left\{x \in M_{i}: g_{i}(x) \geq d-1 / p\right\} .
$$

It follows from the definition of the essential upper bound that

$$
\mathscr{H}^{m}\left(B_{p, i_{0}}\right)>0 \text {. }
$$

Using the inequality $g_{i}\left(T_{e}(x)\right) \geq g_{j}(x)$ for $e \in \mathscr{E}_{i, j}$, we have

$$
\bigcup_{j} \bigcup_{e \in \mathscr{E}_{i, j}} T_{e}\left(B_{p, j}\right) \subset B_{p, i}
$$


Since $\mathscr{H}^{m}\left(B_{p, i}\right)>0$, applying Proposition 9 for all $i$, we have

$$
\mathscr{H}^{m}\left(B_{p, i}\right)=\mathscr{H}^{m}\left(M_{i}\right) \text { for any } p \geq 1 \text {. }
$$

It means that for any $i$ the following subset of $M_{i}$

$$
C_{i}=\bigcap_{p} B_{p, i}=\left\{x \in M_{i}: g_{i}(x) \geq d\right\}
$$

has full measure $\mathscr{H}^{m}\left(M_{i}\right)$. Since $d$ is the maximal essential upper bound of $\left\{g_{i}\right\}_{i}$, we have

for $\mathscr{H}^{m}$-almost all $x \in M_{i}$.

$$
g_{i}(x)=d,
$$

\section{Proofs of Theorems}

\subsection{Version of scaling self-similar sets.}

5.1.1. Proof of Theorem 1. It follows from Proposition 3 that given $V \in$ $G(n, n-m)$, then for $\mathscr{H}^{m}$-almost all $a \in J_{V}$,

$$
\overline{\operatorname{dim}}_{B} E_{V, a} \leq \max \left(0, \overline{\operatorname{dim}}_{B} E-m\right) .
$$

For a self-similar set, we always have $\operatorname{dim}_{B} E=\operatorname{dim}_{H} E$ (e.g., see [Fa3]). And thus, we have

$$
\overline{\operatorname{dim}}_{B} E_{V, a} \leq \max (0, s-m) .
$$

Case I: $s=m$. Using (5.1) and the inequality

$$
\operatorname{dim} E_{V, a} \leq \overline{\operatorname{dim}}_{B} E_{V, a}
$$

for $\operatorname{dim}=\operatorname{dim}_{H}, \underline{\operatorname{dim}}_{B}$ and $\operatorname{dim}_{P}$, we obtain the typical value $0=s-m$.

Case II: $s>m$. By Proposition 1, given $t \in \mathbf{Q}$ with $m<t<s$, for $\gamma_{n, n-m}$-a.e. $V \in G(n, n-m)$,

where

$$
\mathscr{H}^{m}\left(\Omega^{t}\right)>0
$$

$$
\Omega^{t}=\left\{a: \operatorname{dim}_{H}[E \cap(V+a)] \geq t-m\right\} \subset P_{V^{\perp}} E \subset V^{\perp} .
$$

Let $f_{1}(a)=\operatorname{dim}_{H}(E \cap(V+a))$. Then $f_{1}$ is measurable. By Proposition 5,

$$
f_{1}\left(S_{i} a\right) \geq f_{1}(a) \text { for all } i \text {. }
$$

As a result,

$$
\cup_{i} S_{i}\left(\Omega^{t}\right) \subset \Omega^{t} \text { with } \mathscr{H}^{m}\left(\Omega^{t}\right)>0,
$$

where $\left\{S_{i}: V^{\perp} \rightarrow V^{\perp}\right\}_{i}$ are similitudes. It follows from Proposition 8 that

$$
\mathscr{H}^{m}\left(\Omega^{t}\right)=\mathscr{H}^{m}\left(P_{V^{\perp}} E\right),
$$

where $P_{V^{\perp}} E=\cup S_{i}\left(P_{V^{\perp}} E\right)$ (Proposition 4).

Letting $t \rightarrow s$, we have

$$
\mathscr{H}^{m}\left\{a: \operatorname{dim}_{H}\left(E_{V, a}\right) \geq s-m\right\}=\mathscr{H}^{m}\left(\bigcap_{t \in \mathbf{Q}, t<s} \Omega^{t}\right)=\mathscr{H}^{m}\left(P_{V^{\perp}} E\right) .
$$

for $\gamma_{n, n-m}$-a.e. $V \in G(n, n-m)$. Also we notice that

$$
\underline{\operatorname{dim}}_{B} E_{V, a}, \operatorname{dim}_{P} E_{V, a} \in\left[\operatorname{dim}_{H} E_{V, a}, \overline{\operatorname{dim}}_{B} E_{V, a}\right] .
$$

Therefore, Theorem 1 is proved by using (5.1)-(5.3). 
5.1.2. Proof of Theorems 2 and 3. Since $\operatorname{dim}_{H}, \underline{\operatorname{dim}}_{B}, \overline{\operatorname{dim}}_{B}$ and $\operatorname{dim}_{P}$ satisfy (C1)-(C3), it is enough to prove Theorem 3. Here by Proposition 5 , the conditions (C1) and (C2) imply that for any $a \in P_{V^{\perp}}(E)=\cup_{i} S_{i}\left(P_{V^{\perp}}(E)\right)$,

$$
f\left(S_{i} a\right) \geq f(a),
$$

where $f(a)=\operatorname{dim} E_{V, a}$ is $\mathscr{H}^{m}$-measurable by (C3) and $\left\{S_{i}\right\}_{i}$ are contracting similitudes of $V^{\perp}$. Therefore, it follows from Corollary 3 that

$$
\operatorname{dim} E_{V, a}=f(a) \stackrel{\text { a.e. }}{=} c
$$

where $c$ is a constant depending on $V$ and $\operatorname{dim}$.

\subsection{Version of graph-directed constructions.}

\subsubsection{Proof of Theorem 1.}

Lemma 2. Suppose $\operatorname{dim}_{H} K_{1}=\cdots=\operatorname{dim}_{H} K_{l}=s$. Given any $V \in G(n, n-m)$, for each $1 \leq i \leq l$ and $\mathscr{H}^{m}$-almost all $a \in P_{V^{\perp}}\left(K_{i}\right)$

$$
\overline{\operatorname{dim}}_{B}\left[(V+a) \cap K_{i}\right] \leq \max (0, s-m) .
$$

Proof. Notice that the irreducible graph-directed sets are always regular, that is, $\operatorname{dim}_{H} K_{i}=\operatorname{dim}_{B} K_{i}$ for each $i$ (Section 3.1 of [Fa3]). Then the lemma follows from Proposition 3.

Case $I: s=m$. Using (5.6) and the inequality

$$
\operatorname{dim}\left[(V+a) \cap K_{i}\right] \leq \overline{\operatorname{dim}}_{B}\left[(V+a) \cap K_{i}\right]
$$

for $\operatorname{dim}=\operatorname{dim}_{H}, \underline{\operatorname{dim}}_{B}$ and $\operatorname{dim}_{P}$, we obtain the typical value $0=s-m$.

Case II: $s>m$.

Lemma 3. If $\operatorname{dim}_{H} K_{1}=\cdots=\operatorname{dim}_{H} K_{l}=s>m$, then for each $j$ and $\gamma_{n, n-m}$ almost all $V \in G(n, n-m)$, we have $\mathscr{H}^{m}\left[P_{V^{\perp}}\left(K_{j}\right)\right]>0$ and

$$
\operatorname{dim}_{H}\left[(V+a) \cap K_{j}\right] \geq s-m
$$

for $\mathscr{H}^{m}$ almost all $a \in P_{V^{\perp}}\left(K_{j}\right)$.

Proof. For $t \in(m, s) \cap \mathbf{Q}$, let

$$
\Omega_{(W, i)}^{t}=\left\{a \in W: \operatorname{dim}_{H}\left[\left(W^{\perp}+a\right) \cap K_{i}\right] \geq t-m\right\} .
$$

It follows from Proposition 1 that for $\gamma_{n, m}$-almost all $W \in G(n, m)$,

$$
\mathscr{H}^{m}\left(\Omega_{(W, i)}^{t}\right)>0, \forall t \in(m, s) \cap \mathbf{Q} \text { and } i .
$$

Let

$$
\Pi=\left\{W \in G(n, m): \mathscr{H}^{m}\left(\Omega_{(W, i)}^{t}\right)>0, \forall t \in(m, s) \cap \mathbf{Q} \text { and } i\right\} .
$$

Then

$$
\gamma_{n, m}[G(n, m) \backslash \Pi]=0 .
$$

Given $W^{*} \in \Pi$ and $j^{*} \in \mathbf{N} \cap[1, l]$, let

$$
\Xi=\Xi\left(W^{*}, j^{*}\right)
$$

For $(W, i) \in \Xi$ and $t \in(m, s) \cap \mathbf{Q}$, let

$$
\Omega_{(W, i)}^{t}=\left\{a \in W: \operatorname{dim}_{H}\left[\left(W^{\perp}+a\right) \cap K_{i}\right] \geq t-m\right\} .
$$


For any edge $e$ from $\left(W_{1}, i_{1}\right)$ to $\left(W_{2}, i_{2}\right)$ and any $x \in \Omega_{\left(W_{2}, i_{2}\right)}^{t}$, by Proposition 7 , we have

$$
\operatorname{dim}\left[\left(W_{1}^{\perp}+T_{e}(x)\right) \cap K_{i_{1}}\right] \geq \operatorname{dim}\left[\left(W_{2}^{\perp}+x\right) \cap K_{i_{2}}\right] \geq t-m,
$$

which implies

Now, we have

$$
T_{e}\left(\Omega_{\left(W_{2}, i_{2}\right)}^{t}\right) \subset \Omega_{\left(W_{1}, i_{1}\right)}^{t}
$$

(1) By (3.10), $\left\{P_{W} K_{i}\right\}_{(W, i) \in \Xi}$ are compact sets satisfying

$$
K_{(W, i)}=\bigcup_{\left(W^{\prime}, i^{\prime}\right)} \bigcup_{e \in \Gamma_{(W, i),\left(W^{\prime}, i^{\prime}\right)}} T_{e}\left[K_{\left(W^{\prime}, i^{\prime}\right)}\right],
$$

and $\Xi$ is irreducible (Proposition 6).

(2) For any $(W, i) \in \Xi$,

$$
\Omega_{(W, i)}^{t} \subset P_{W} K_{i} .
$$

For any edge $e$ from $\left(W_{1}, i_{1}\right)$ to $\left(W_{2}, i_{2}\right)$,

$$
T_{e}\left(\Omega_{\left(W_{2}, i_{2}\right)}^{t}\right) \subset \Omega_{\left(W_{1}, i_{1}\right)}^{t} .
$$

(3) Since $W^{*} \in \Pi$,

$$
\mathscr{H}^{m}\left(\Omega_{\left(W^{*}, j^{*}\right)}^{t}\right)>0 .
$$

Applying (1)-(3) to Proposition 9, we have

$$
\mathscr{H}^{m}\left(\Omega_{(W, i)}^{t}\right)=\mathscr{H}^{m}\left(P_{W} K_{i}\right)
$$

for any $(W, i) \in \Xi$ and $t \in(m, s) \cap \mathbf{Q}$. Letting $t \rightarrow s$, since $\left(W^{*}, j^{*}\right) \in \Xi$, we have

$$
\mathscr{H}^{m}\left\{a \in W^{*}: \operatorname{dim}_{H}\left[\left(\left(W^{*}\right)^{\perp}+a\right) \cap K_{j^{*}}\right] \geq s-m\right\}=\mathscr{H}^{m}\left(P_{W^{*}} K_{j^{*}}\right)
$$

for any $W^{*} \in \Pi$ and $1 \leq j^{*} \leq l$. The lemma is proved since $\gamma_{n, m}[G(n, m) \backslash \Pi]=0$.

Now notice that for any set $A$,

$$
\underline{\operatorname{dim}}_{B} A, \operatorname{dim}_{P} A \in\left[\operatorname{dim}_{H} A, \overline{\operatorname{dim}}_{B} A\right] .
$$

Therefore, Theorem 1 is proved by Lemma 2, 3 and (5.8).

5.2.2. Proof of Theorems 2 and 3. Since $\operatorname{dim}_{H}, \underline{\operatorname{dim}}_{B}, \overline{\operatorname{dim}}_{B}$ and $\operatorname{dim}_{P}$ satisfy (C1)-(C3), it is enough to prove Theorem 3. Let

$$
g_{(W, i)}(x)=\operatorname{dim}\left[(W+x) \cap K_{i}\right] .
$$

Then $g_{(W, i)}(x)$ is $\mathscr{H}^{m}$-measurable by (C3).

By Proposition 7, the conditions (C1) and (C2) imply that given an edge $e$ from $\left(W_{1}, i_{1}\right) \in \Xi$ to $\left(W_{2}, i_{2}\right) \in \Xi$, then for any $x \in P_{W_{2}} K_{i_{2}}$,

$$
g_{\left(W_{1}, i_{1}\right)}\left(T_{e}(x)\right) \geq g_{\left(W_{2}, i_{2}\right)}(x) .
$$

Therefore, it follows from (C3) and Corollary 4 that

$$
\operatorname{dim}\left[(W+x) \cap K_{i}\right]=g_{(W, i)}(x) \stackrel{\text { a.e. }}{=} c,
$$

where $c$ is a constant depending on $W$ and dim, This completes the proof of Theorems 2 and 3 . 


\section{References}

[BP] Benjamini, I., and Y. Peres: On the Hausdorff dimension of fibres. - Israel J. Math. 74, 1991, 267-279.

[C] CsöRnYeI, M.: On planar sets with prescribed packing dimensions of line sections. - Math. Proc. Cambridge Philos. Soc. 130:3, 2001, 523-539.

[Fa1] Falconer, K. J.: The geometry of fractal sets. - Cambridge Univ. Press, 1985.

[Fa2] Falconer, K. J.: Sets with large intersections. - J. London Math. Soc. 49, 1994, 267-280.

[Fa3] Falconer, K. J.: Techniques in fractal geometry. - John Wiley, 1997.

[FJ] Falconer, K. J., and M. JÄrvenpäÄ: Packing dimensions of sections of sets. - Math. Proc. Cambridge Philos. Soc. 125:1, 1999, 89-104.

[FJM] Falconer, K. J., M. JÄrvenpäÄ, and P. Mattila: Examples illustrating the instability of packing dimensions of sections. - Real Anal. Exchange 25:2, 1999/00, 629-640.

[FM] Falconer, K. J., and P. Mattila: The packing dimension of projections and sections of measures. - Math. Proc. Cambridge Philos. Soc. 119:4, 1996, 695-713.

[Fe] Federer, H.: Geometric measure theory. - Springer-Verlag, 1969.

[Ha] Hawkes, J.: Some algebraic properties of small sets. - Quart. J. Math. Oxford Ser. (2) 26, 1975, 195-201.

[Hu] Hutchinson, J. E.: Fractals and self similarity. - Indiana Univ. Math. J. 30, 1981, 713-747.

[JM] JÄrvenpä̈̈, M., and P. Mattila: Hausdorff and packing dimensions and sections of measures. - Mathematika 45:1, 1998, 55-77.

[K] Kahane, J. P.: Sur la dimension des intersections. - In: Aspects of mathematics and its applications, North-Holland Math. Library 34, North-Holland, Amsterdam, 1986, 419-430.

[KP] Kenyon, R., and Y. Peres: Intersecting random translates of invariant Cantor sets. Invent. Math. 104, 1991, 601-629.

[L] LI, J. F.: On the dimensions of intersections of Sierpinski carpet with lines. - Master's thesis, Wuhan Univ., 1997.

[LXZ] LiU, Q. H., L. F. XI, and Y. F. ZhaO: Dimensions of intersections of Sierpinski carpet and lines with rational slopes. - Proc. Edinb. Math. Soc. (2) 50:2, 2007, 411-427.

[M] Marstrand, J. M.: Some fundamental geometrical properties of plane sets of fractional dimensions. - Proc. London Math. Soc. (3) 4, 1954, 257-302.

[Mat1] Mattila, P.: Hausdorff dimension, orthogonal projections and intersections with planes. Ann. Acad. Sci. Fenn. Ser. A I Math. 1, 1975, 227-244.

[Mat2] MattilA, P.: Integralgeometric properties of capacities. - Trans. Amer. Math. Soc. 266, $1981,539-554$.

[Mat3] Mattila, P.: On the structure of self-similar fractals. - Ann. Acad. Sci. Fenn. Ser. A I Math. 7, 1982, 189-195.

[Mat4] MatTilA, P.: Hausdorff dimension and capacities of intersections of sets in $n$-space. - Acta. Math. 152, 1984, 77-105.

[Mat5] Mattila, P.: Geometry of sets and measures in Euclidean spaces. - Cambridge Univ. Press, 1995.

[MM] Mattila, P., and R. D. Mauldin: Measure and dimension functions: measurability and densities. - Math. Proc. Cambridge Philos. Soc. 121:1, 1997, 81-100.

[MW] Mauldin, R. D., and S. C. Williams: Hausdorff dimension in graph directed constructions. - Trans. Amer. Math. Soc. 309:1-2, 1988, 811-839.

[S] Schief, A.: Separation properties for self-similar sets. - Proc. Amer. Math. Soc. 122, 1994, $111-115$.

Received 15 September 2009 